\title{
Simple Shape-from-Shading for Human Surface Measurement
}

\author{
Harvey MITCHELL* \\ Civil Surveying and Environmental Engineering, University of Newcastle, Newcastle, Australia
}

\begin{abstract}
Shape-from-shading (SFS) suggests itself as suitable technique for three-dimensional body shape measurement, because it depends on a lack of optical texture on the object, and this lack of texture is a characteristic found across much of the human body. Measurement by SFS is also appealing because it requires just a single image. However, SFS does not easily produce accurate and reliable results. The goal of the work reported in this paper has been to seek three-dimensional results of adequate accuracy for certain human measurement applications by using an approach emphasising low cost and a lack of complication. This work was initiated by a desire to measure extracted and live human teeth, but trials have also used human body areas. Results show some limitations to the current approach, but they are encouraging and the concept of achieving shape measurement goals without complication is valid and modifications to the approach will continue.
\end{abstract}

Keywords: shape-from-shading, tooth measurement, human back measurement

\section{Introduction}

Shape-from-shading (SFS) is an attractive technique for three-dimensional body shape measurement because its fundamental requirement is a lack of optical texture, a characteristic which can be found over much of the human body. The need for a lack of texture contrasts it with photogrammetry whose success is contingent on the availability of plenty of optical texture. This work was stimulated by a desire to measure human teeth, especially for monitoring changes in children's teeth, by utilising the clean plain surfaces which characterise young teeth.

SFS has a second advantage in the simplicity and low cost at the data collection stage, as it requires nothing more than a single image from any camera with a flash in a dark environment. The instant imaging avoids problems caused by human movement during the imaging, and this can provide SFS with an advantage over scanning.

Given the low cost of digital cameras, SFS is appealing. But, SFS does have. The crucial element of the work which is reported here is to seek acceptable solutions to the problems of SFS, with the aim of using SFS for three-dimensional surface-shape measurement technique for some body measurement situations where the use of more sophisticated approaches is not needed. Algorithm development has required extensive work in many applications of the SFS technique, but the emphasis here is on cheapness and simplicity for adequate output.

\section{Concepts of Shape-From-Shading}

The basic concept behind SFS is simple: under certain suitable lighting conditions, the strength of the reflections of a light source from a plainly textured surface are related to the surface slopes across the object. This can enable the full surface shape of the object to be reconstructed.

More specifically, for any surface element on the object, the intensity of a reflected ray is related to the angle between the reflected ray and the ray from the source. If the light source is coincident with a camera lens centre, then the recorded intensity of light reflection can be used to calculate the angles between the surface element and the ray from the lens. This angle can easily be converted to an angle relative to the camera's axis, thus providing the gradient of the surface element relative to the sensor's optical axis. An array of such gradients from a single image may then enable the surface shape to be reconstructed.

The fundamental equation is simple provided that the surface is subject to Lambertian reflection (by which the light hitting the surface is partially absorbed and partially transmitted, but, more importantly, the reflection is distributed equally in all directions).

* harvey.mitchell@newcastle.edu.au; +61-2-49216055; www.newcastle.edu.au 
According to Zhang et al. [1], for pure Lambertian reflection, the reflectance level is given by:

$$
R=A \rho \cos \theta
$$

where $R$ is the reflectance level recorded at a point on the image,

$A$ is the strength of the light source,

$\rho$ is the albedo of the surface, and

$\theta$ is the angle between the surface normal and the source direction at the imaged point.

For a uniformly textured surface, we can assume that $A \rho$ is constant.

The goal of SFS is to deduce values of $\theta$ from an array of values of $R$ across an image, in order to convert them to surface gradients, and to then transform the surface gradients into surface shape.

\section{Difficulties with Shape-From-Shading}

The prospective user of SFS for body shape measurement needs to be aware of the difficulties which may be faced in practical application of the equation above. The difficulties can be listed, in what is probably an increasing order of severity, as follows:

1. SFS requires good quality imagery, and it requires that the surface must be illuminated by an appropriate light source. To simplify the geometry, the light source should be close to the imaging lens. In practice, the flash on a normal camera can be used as the light source and it may be seen to be sufficiently close to the lens if the camera is not close to the object.

2. Scale is not normally obtainable from SFS because it uses only one image. Multiple images can be utilised [2], but this may is regarded as unnecessarily complicated in this application.

3. Regions of bright specular reflection can interrupt the solution, and thus disrupt the surface shape solution significantly. One solution is to avoid surface gradients close to zero by taking imagery which is oblique to the surface.

4. The solution for the surface normal direction from the reflectance data is a function of $A$ and $\rho$, or more specifically their product, $A \rho$, and $A \rho$ may not be known, especially if the imagery is oblique to the surface, as recommended at 3 .

5. SFS methods have difficulties if the surfaces are not of even optical texture, in which case $A \rho$ will vary. However, the lack of optical texture should not be a problem in many cases of measuring suitable plain surfaces such as found on the human body in many cases. But in the case of human body measurement it means that measurement cannot easily be carried out over both skin and clothing on the same patient.

6. Following on from 5 , the value of $A \rho$ can vary from object to object, or even from one image to another image of the same object. The relationship between reflectance level and albedo should be held constant, by using standard photographic conditions or fixed camera settings, so automated digital image processing can create difficulties. Cameras with manually controllable aperture rating and exposure time, or equivalent parameters, can help control the albedo-reflectance relationship.

7. The solution for the slope from the recorded image intensity involves the inverse cosine, by Equation (1). The cosine is close to unity when the slope angle is close to zero, i.e. when the camera axis is approximately normal to the object surface, so the cosine can vary more slowly than the angle, and the solution for the angle can be insensitive. Oblique angled imagery, as suggested at 3 , can reduce this problem. As well, the solution for the slope angle is sensitive to noise in the reflectance value. Indeed, noise in the reflectance value can cause the cosine of the slope to appear to be greater than unity. The noise may be reduced - but often incompletely and inadequately - by filtering. Averaging multiple images to reduce noise introduces other disadvantages when simplicity is a goal.

8. SFS indicates the direction of the surface normal, and from the surface normal directions surface shape then need to be reconstructed. Thus, SFS could be described as slope-from-shading rather than shape-from-shading. Integration of gradients to compile a complete surface is not necessarily straight-forward. Integration can also mean the accumulation of any errors occurring in some regions of the object.

9. More than anything else, the direction of the surface normal must be defined by two quantities, whereas the reflectance provides only a single data value, and a solution is apparently impossible. In practice, assumptions can often be made about the surface characteristics, such as that the direction of the surface normal alters smoothly, and without discontinuities. Even without discontinuities, the problem can be difficult if there is uncertainty of the sign of the cosine in Equation (1) when there are changes in local object surface slope around zero. Clearly, SFS has difficulties if the surfaces are convoluted in shape. Some areas of the human body are more complex than others. 


\section{Model Testing: Plane Surfaces}

The validity of the model given by Equation (1) can be demonstrated using objects of known shape. For example, flat objects (paper of various light colours) have been imaged using a Flexiscope Piccolo intraoral dental camera, which has in-built light sources close to the lens. The camera was set to obtain 24-bit monochrome images, at $760 \times 576$ pixels, but the images were reduced to 8-bit grey-levels and to $144 \times 96$ pixels to permit simpler manipulation during testing stages. Being an intraoral dental camera its lens size and the distances from the light sources to the lens are small relative to the objects being measured.

The Flexiscope camera has a short focal length and high distortion levels. The camera focal length (which is needed to convert the deduced angles $\theta$ from angles relative to the outgoing ray to angles relative to the camera axis) and distortion characteristics were deduced in a multi-image photogrammetric solution of an object about $10 \mathrm{~mm} \times 10 \mathrm{~mm} \times 10 \mathrm{~mm}$ in size [3], with photogrammetric processing by version 3.1 of PhotoModeler software [4]. Figure 1 shows the original image of a flat white card, which was at $57^{\circ}$ to the axis of the camera, while figure 2 shows the surface shape deduced using equation (1), via nothing more complicated than a Microsoft Excel spread-sheet calculation, with some edge effects removed. The slope of that surface was calculated to be $60^{\circ}$. Good results for a flat surface are not proof of the algorithm's legitimacy, but an encouraging demonstration of the approach which is being taken.



Fig. 1. Observed reflectance levels of a flat white card recorded by a Flexiscope Piccolo intra-oral dental camera. The image has been reduced to 144 pixels $x 108$ pixels for processing.

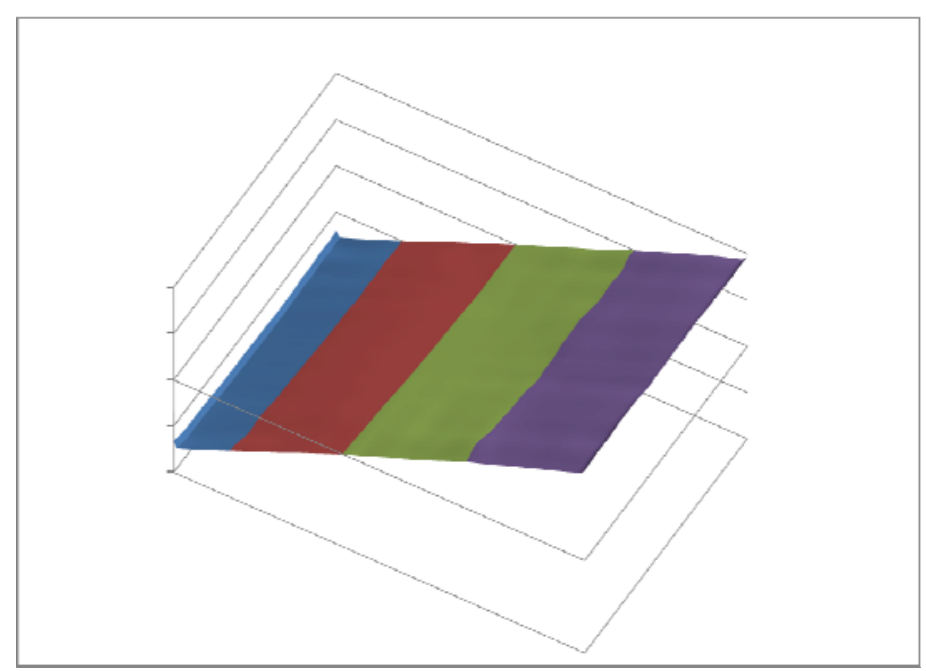

Fig. 2. Resulting surface shape, deduced using at Equation (1) with the image at figure 1, derived via a Microsoft Excel spread-sheet calculation. 


\section{Case Study: Extracted Human Tooth}

The studies described were initiated by a desire to monitor changes in tooth surface shape, to replace more laborious replication and mechanical measurement methods in a dental erosion study $[5,6,7]$. The figures below show, first, a suitable flash image of an extracted tooth (figure 3 ), second, the surface gradients as subsequently deduced (figure 4) and third, the results of integrating the gradients to form the surface shape (figure 5). Accuracies are untested at this stage.

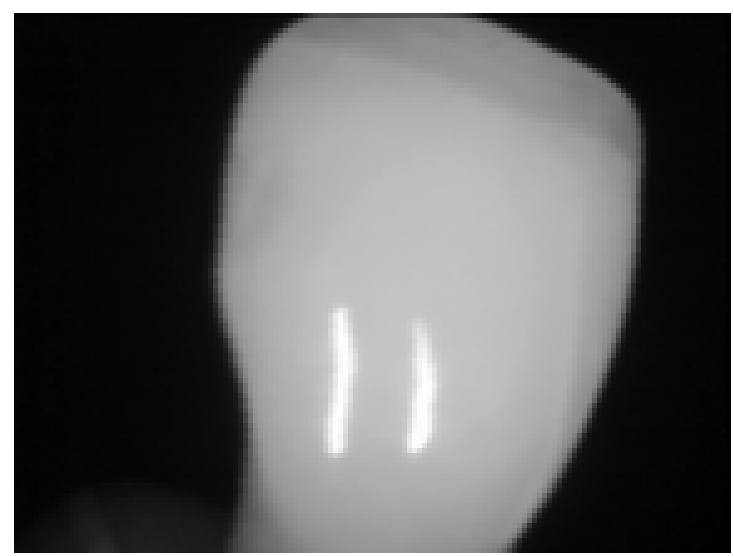

Fig. 3. Intraoral camera image of extracted human tooth. Direct light reflections appear on part of the tooth.

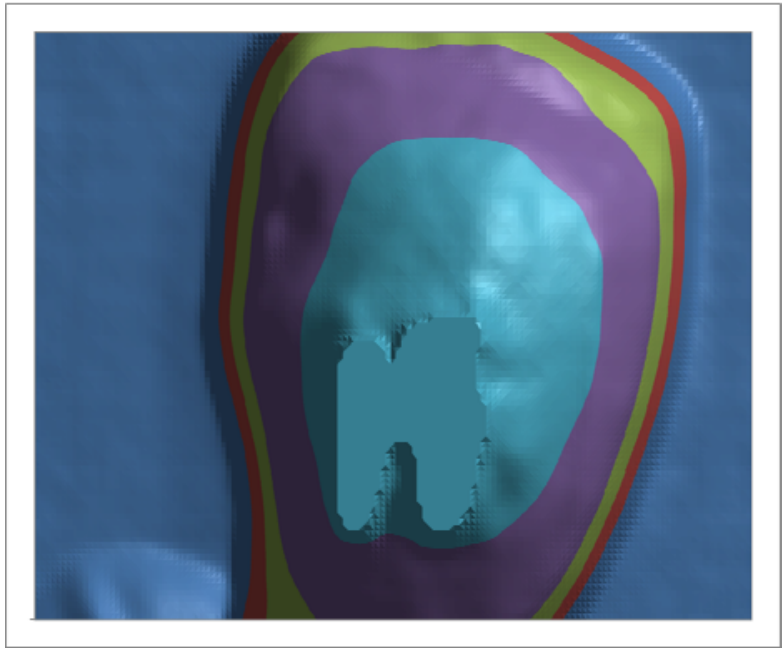

Fig. 4. The reflectance values derived from Figure 7, with areas of bright reflectance removed by simple thresholding of the reflectance values.

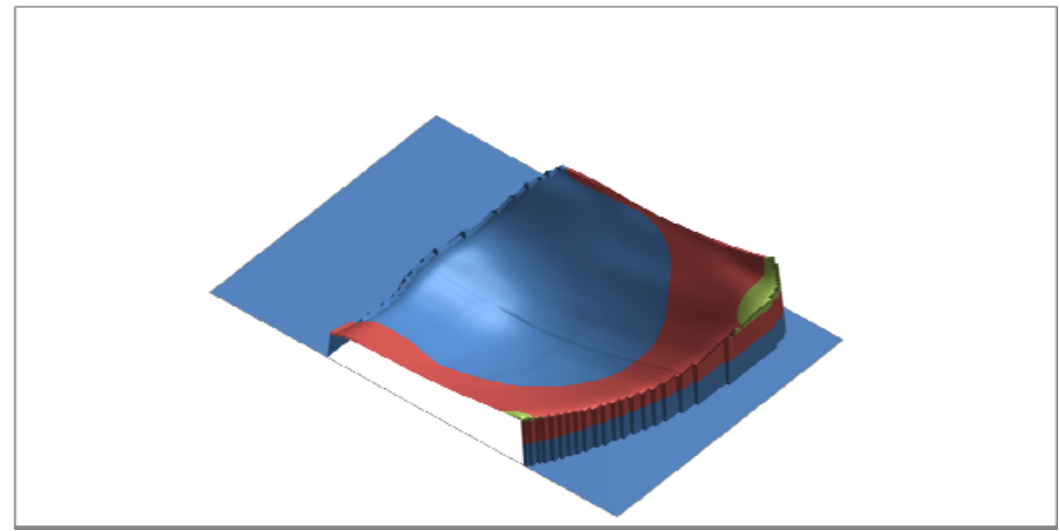

Fig.5. The results of integration of gradients to form the surface profile. Some problems are caused by the elimination of the bright spots, which cause an accumulated integration error. 


\section{Case Study: Live Human Teeth}

The figures in this case show a flash image within the mouth (figure 6), the deduced surface gradients (figure 7) and finally the results of integration of the gradients to form the surface shape (figure 8). Complications occur in areas of convoluted variation in the slope angles. Accuracies are again untested.

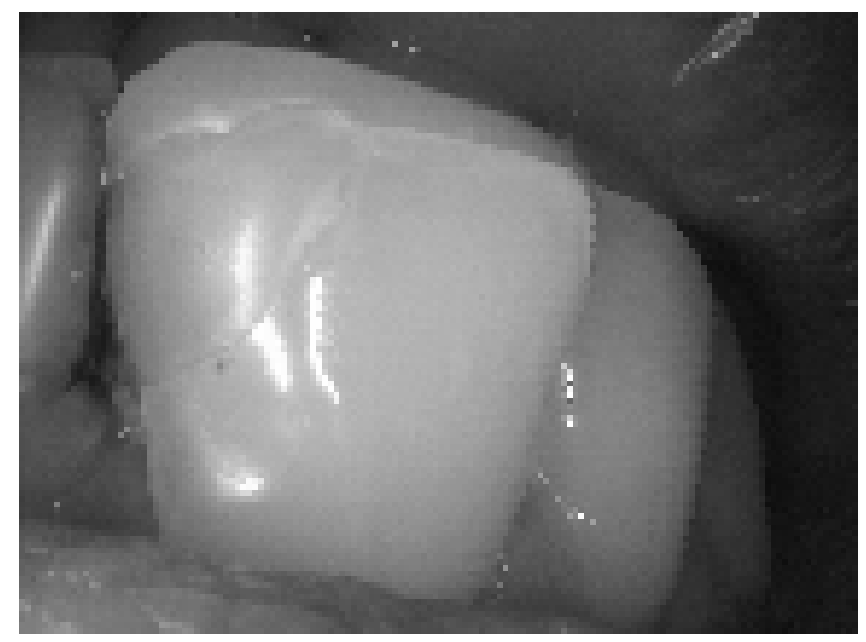

Fig. 6. Intraoral camera image of human teeth.

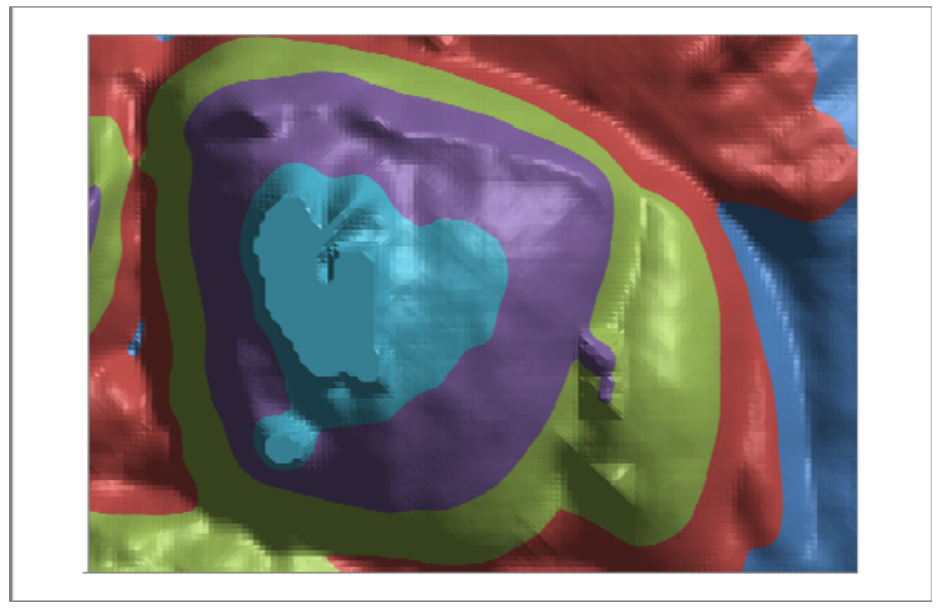

Fig. 7. The reflectance values derived from Figure 7, with areas of bright reflectance removed by simple thresholding of the reflectance values.

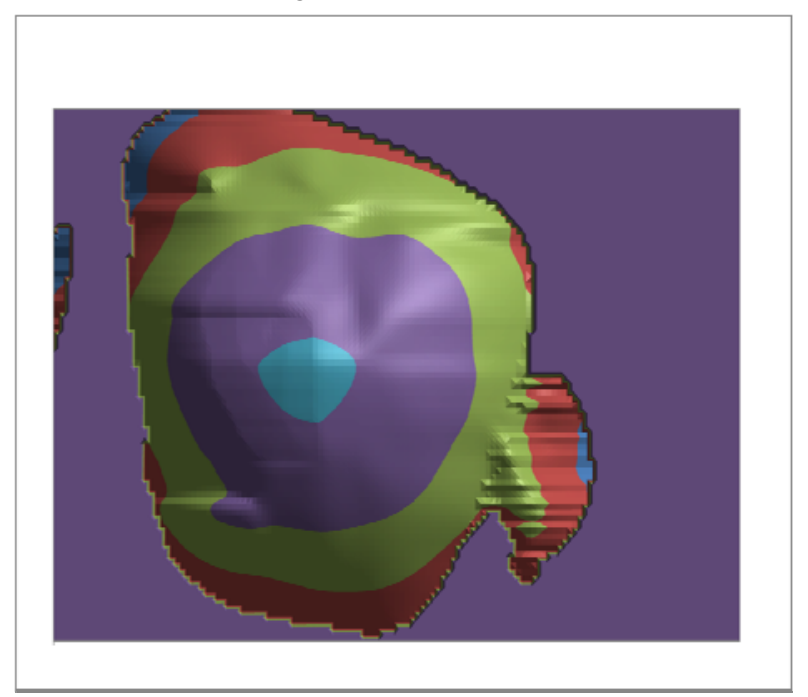

Fig. 8. The results of integration of gradients to form the surface profile. As with the extracted tooth, problems are caused by the elimination of the bright spots, which cause an accumulated integration error. 


\section{Case Study: Human Back}

The possibility of using SFS to determine body shape was investigated with imagery of a human back, this time using a Canon EOS30D single-lens-reflex digital camera with a $28 \mathrm{~mm}$ Canon lens, with the camera's built-in flash as the light source. The camera was set to obtain 24-bit monochrome images, at $3504 \times 2336$ pixels, but they were reduced to 8-bit grey-levels. As before, the image size was reduced, this time to $144 \times 108$ pixels for simpler manipulation during early stages of this investigation. The figures below show the stages in the data collection and processing: a flash image of the back (figure 9), the recorded reflectance levels for the central row of from figure 9, are shown in figure 10. The reflectance levels range from almost zero towards 256 for 8-bit grey-levels.

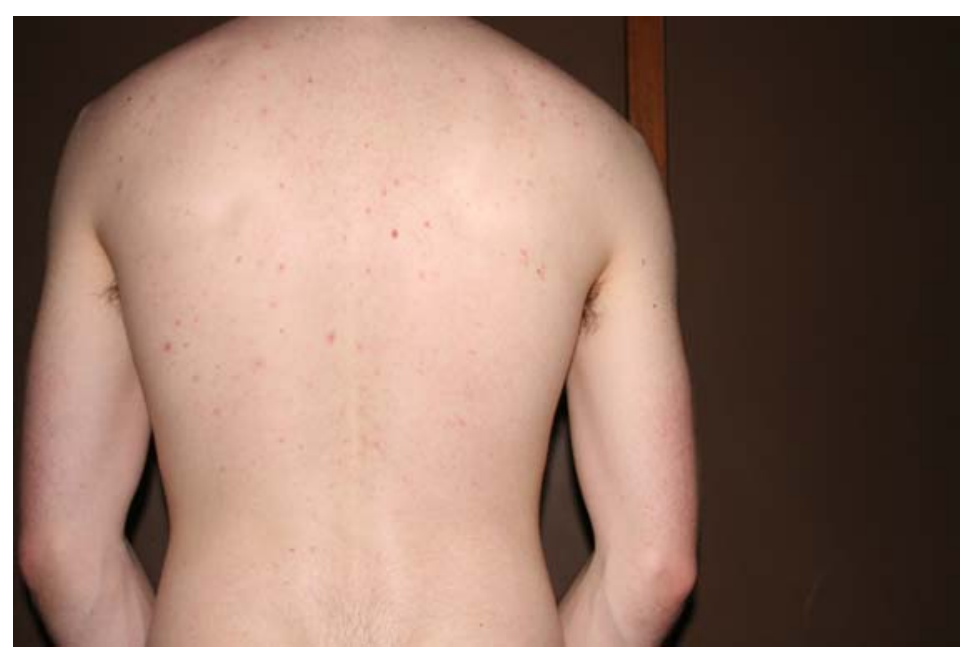

Fig. 9. Flash image of a human back

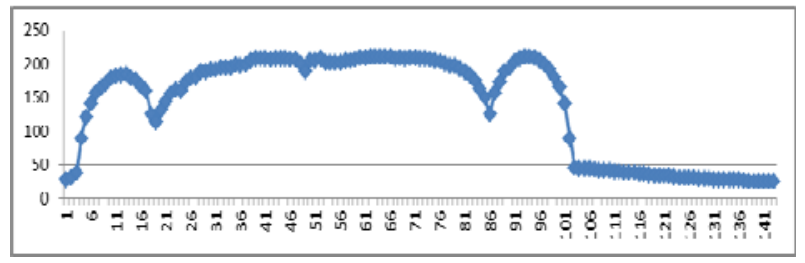

Fig. 10. The recorded reflectance values for the central row of from figure9 when converted to monochrome.

The angles, $\theta$, between the surface normal and the direction of the light source direction are shown in figure 11; the results of integration of the gradients to form the surface shape are in figures 12 and 13.



Fig. 11. The angles, $\theta$, between the surface normal and the direction of the light source direction. 


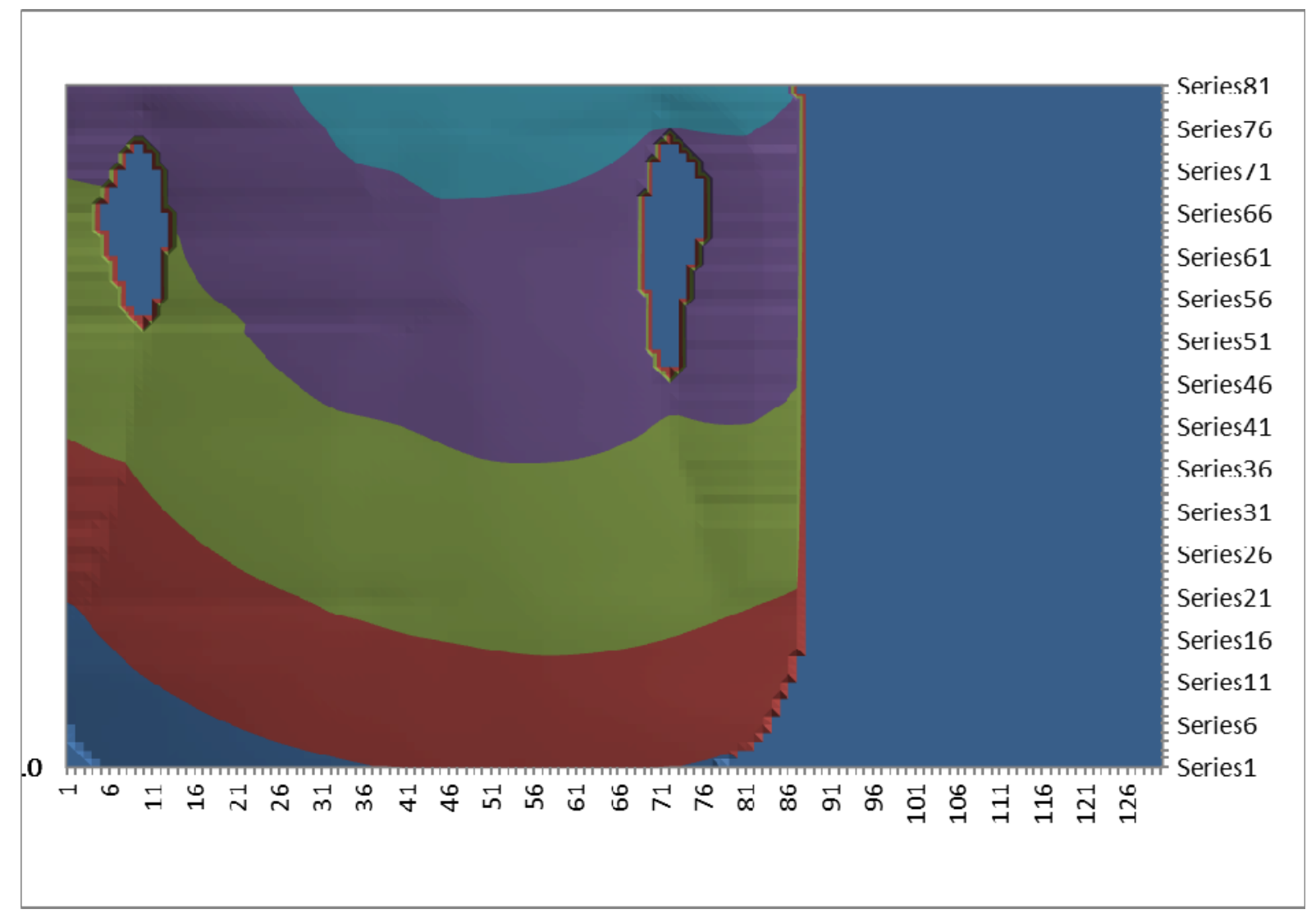

Fig. 12. The results of integration of surface gradients to form the full surface shape. (The arms have not been included in the integration. Problems can be seen to occur in regions of complicated surface shape.

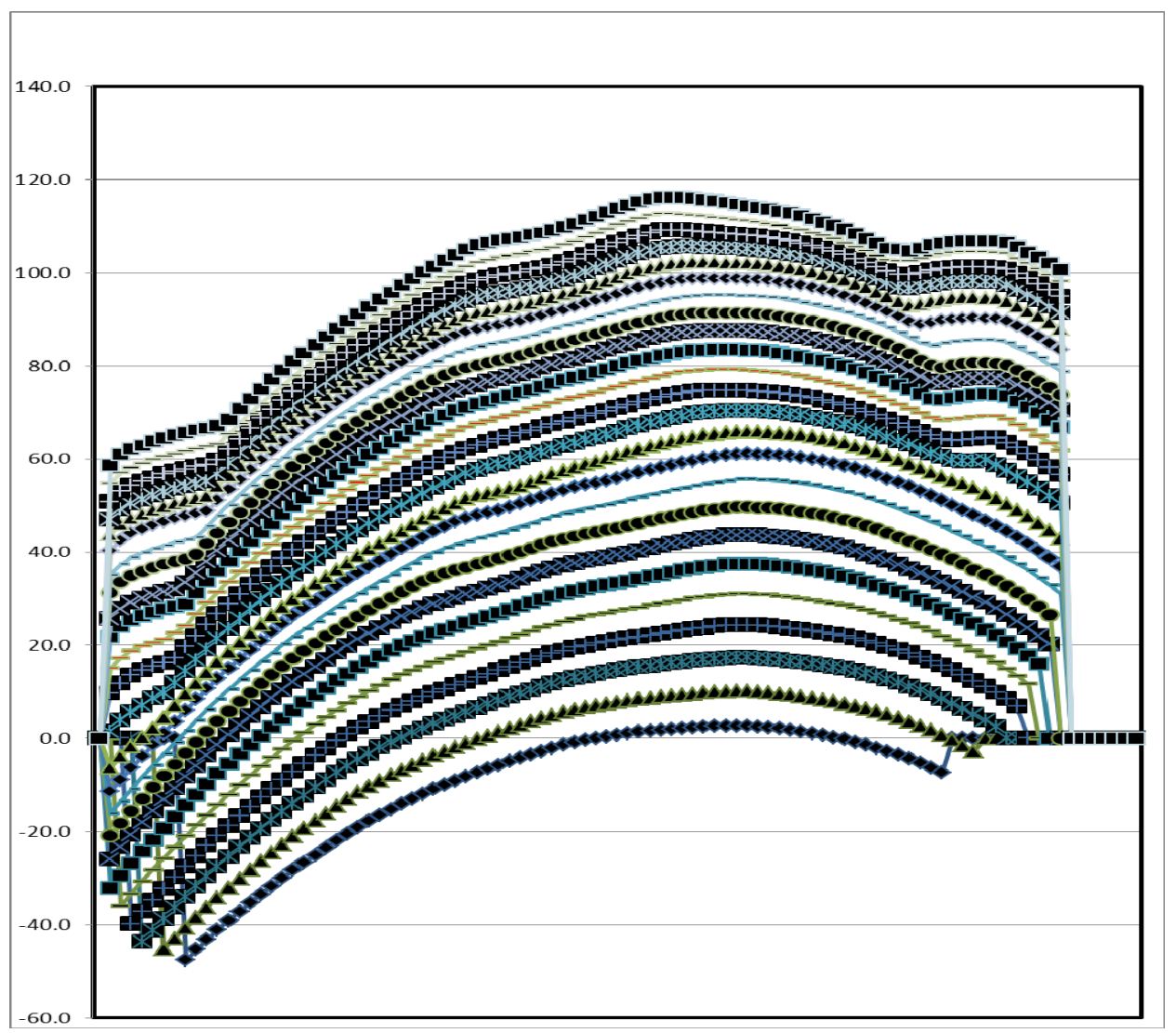

Fig. 13. Profiles confirm that distances around the body should be measurable for apparel fitting. 
Figures 12 and 13 both reveal how the indentation around the spine has been modelled.

As suggested earlier, problems occur in regions of complicated surface shape. While the reflectances in figure 9 look sensible to human examination, errors appear in the depiction of the three-dimensional surface appear when the gradients are integrated. Examination of the reflectance levels and the reflectance angles shows that errors occur primarily in areas of convoluted variation in the slope angles. It will be noticed that flaws of this nature do not occur in the smooth areas nor do they occur in the deduced shape of the plane object in figure 2. Figure 13 shows the complicated variation in reflectance level across an area ( 26 pixels wide) where reflectance levels create a local maximum.

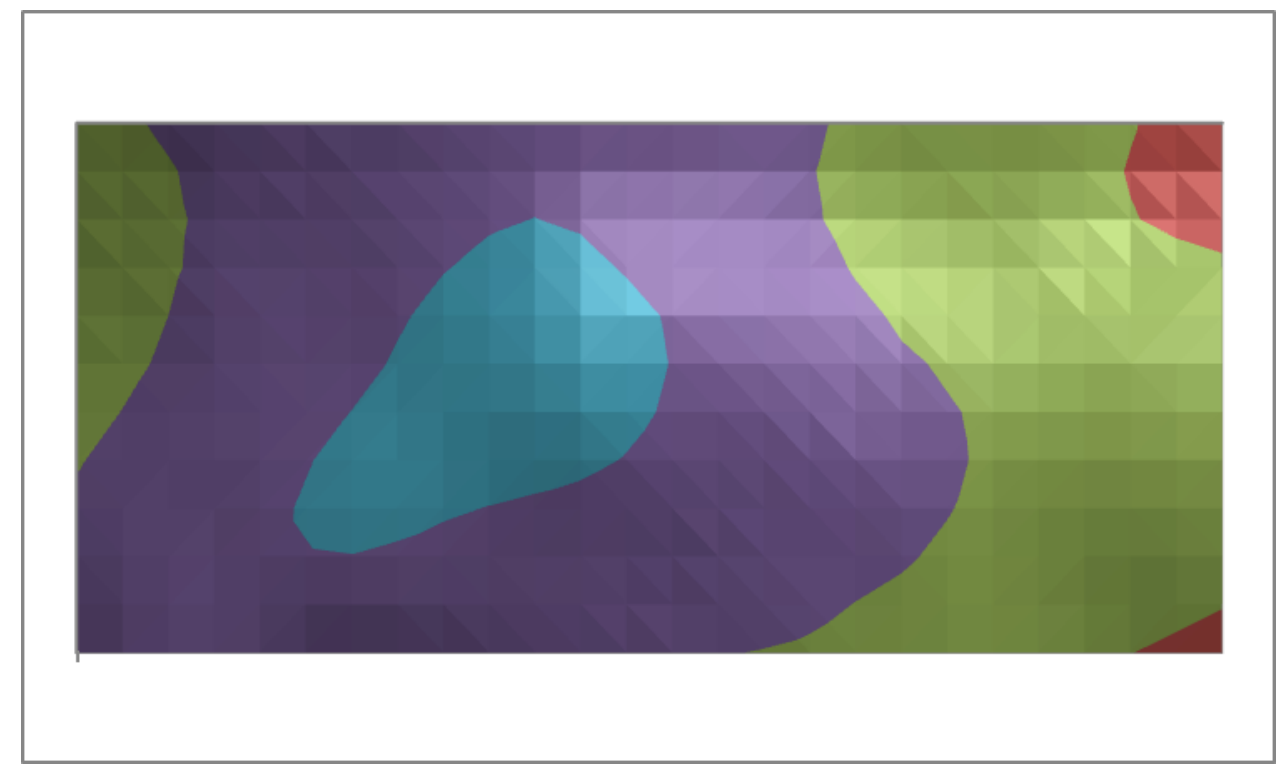

Fig. 14. An example of complicated reflectance variation, occurring near the shoulder blade of the image in figure 9 , is indicated by a local maximum.

The accuracies of the final shape are completely untested at this stage. But even with its flaws, the final product can be seen to provide an outline of body shape which has been obtained extremely simply. Figure 13 suggests that distances around the body profiles should be measurable for applications such as apparel fitting.

\section{Discussion}

The results reported here are of an investigation into the feasibility of using SFS based on Equation (1) using as many simplifications as possible, to make the procedure appealing in those situations when expense, especially of equipment, cannot be justified, perhaps in scoliosis screening or widespread consumer sizing for apparel fitting. The only equipment used has been a camera with a flash or fixed light source, and all calculations (and diagrams) have been derived using only spread-sheet calculations.

It must be emphasised that the surface shapes created by integration of the gradients provides quantitative information which is not provided by the original image, even though at first glance, the reflectance levels in original images seem to be usable to indicate surface shape.

\section{Conclusions}

Results obtained so far are encouraging, because progress towards the goals has been made. Problems in areas of convoluted surface shape, as anticipated in Section 3 of the paper, may be relieved by more oblique imaging directions, avoiding imaging which faces the surface at an angle too close to $90^{\circ}$.

The goal is to develop utilise simple and low data capture with uncomplicated processing to measure objects which are defined by simple shapes, such as may be encountered in some body areas, and eventually live children's teeth as well. 


\section{References}

1. Zhang, R., Tsai, P-S., Cryer, J.E., Shah, M., (1999): "Shape from shading: a survey", IEEE Transactions on Pattern Analysis and Machine Intelligence, Vol. 21, No. 8, pp.690-706.

2. Heipke, C. (1992): "Integration of digital image matching and multi-image shape from shading", International Archives of Photogrammetry and Remote Sensing, (Proceedings of ISPRS Congress, Washington), Vol. 29, No. B3, pp.832-841.

3. Mitchell, H. L. and Chadwick, R.G., (2008): "Challenges of Photogrammetric Intra-Oral Tooth Measurement", The International Archives of the Photogrammetry, Remote Sensing and Spatial Information Sciences (ISSN 1682-1750) Vol. 37, No. B5: pp.779-782.

4. Photogrammetric software "PhotoModeler" (accessed 2012): http://www.photomodeler.com/

5. Chadwick, R.G., Mitchell, H.L., Cameron, I., Hunter, B., \& Tulley, M., (1997): "Development of a novel system for assessing tooth and restoration wear", Journal of Dentistry, Vol. 25, No. 1, pp.41-47.

6. Mitchell, H.L., Chadwick, R.G., Ward, S. \& Manton, S.L., (2003): "A comprehensive system for detecting erosion on children's teeth", Medical \& Biological Engineering and Computing, Vol. 41, No. 4, pp.464-469.

7. Chadwick, R.G., Mitchell, H.L., Manton, S.L., Ward, S., Ogston, S. \& Brown, R., (2005): "Maxillary Incisor Palatal Erosion - No correlation with dietary variables?", Journal of Clinical Pediatric Dentistry, Vol. 29, No. 2, pp.157-163. 